hence its name. By invoking a small number of characters (especially ill-defined ones) one can construct many contradictory taxa: for example, cyanobacteria can be clustered with the extreme halophiles to the exclusion of other eubacteria on the basis of ferredoxin sequences; or, streptomycetes can be grouped with methanogens on the basis of their having coenzyme F420.

Lake's explanation of the rRNA-based tree (Fig.1a) is that it must be an artefact of the treeing procedure (such procedures have a tendency to group rapidly evolving lines). Although such artefacts do occur, they obviously do not occur in every case involving unequal evolutionary rates. A thorough analysis indicates that the branching order in Fig. $1 a$ does not reflect such an artefact? ${ }^{\text {. }}$.

Returning to the semantic issue, regardless of whether the archaebacteria are joined to the two other kingdoms by a single lineage (Fig.1a) or by two separate lineages (Fig.1b), archaebacteria is a taxon of demonstrated predictive and organizational value ${ }^{7}$. It groups together prokaryotes with many characteristic phenotypic features, including composition of membranes, cell walls, rRNAs and tRNAs ${ }^{10}$. Lake's taxa, on the other hand, spread such organisms among several kingdoms (as indicated by the solid lines in the Fig. 1), intermixed with organisms not sharing their common characteristics. Whereas the grouping 'archaebacteria' easily rationalizes almost all of what is known about these organisms, the Lake taxa are inconsistent with much of the phenotypic evidence and all of the sequence evidence bearing on the issue.

CARL R. Woese

Department of Genetics and Development, University of Illinois,

Urbana, Illinois 61801, USA

NORMAN R. PACE Gary J. Olsen

Department of Biology,

Indiana University,

Bloomington, Indiana 47405, USA

1. Lake, J.A., Henderson, E.. Clark, M.W. \& Oakes, M. Proc. natn. Acad. Sci. U.S.A. 81, 3786 (1984).

2. Lake, J.A. et al. Proc. natn. Acad. Sci. U.S.A. 82, 3716 (1985)

3. Lake, J.A. Nature 319,626 (1986)

4. Zillig, W. Nature 320, 220 (1986)

Lederer, H. Nature 320, 220 (1986)

Wilson, A.C. Scient. Am. 253(4), 164 (1985)

7. Woese, C.R. \& Olsen, G.J. System. Appl. Microbiol. (in the press)

8. Stöffler-Meilicke, M., Böhme, C., Strobel, O., Böck, A.\& Stöffler. G. Science 231, 1306 (1986)

9. Cammarano, P., Teichner, A. \& Londei, P. System. Appl. Microbiol. (in the press).

10. Wocse, C R \& Wolfe, R.S. (eds) The Bacteria Archaebacteria Vol. 8 (Academic, New York, 1985).

\section{Link between lamins and intermediate filaments}

SIR-Recently McKeon et al. ${ }^{\prime}$ reported in Nature an exciting result for cell biologists. Lamins, the major proteins of the nuclear envelope, show a striking sequ- ence and structural homology with the various proteins of the large family of cytoplasmic intermediate filaments (IFs). The complementary DNA sequence of lamins $A$ and $C$ predicts, however, an insert of 42 amino-acid residues in a coiled-coil domain highly conserved in length in all IF proteins (reviewed in ref. 2). Inspecting the reported lamin sequence I found that this insert starts precisely at a point of a common intron position previously found in several IF genes ${ }^{2}$. The position of most introns of IF genes seems not to be related to the structural subdomains of the proteins ${ }^{2}$. A similar situation was found also for myosin, another fibrous protein built from coiled-coils ${ }^{3}$. Thus, the position of the lamin insert is particularly interesting as it may indicate the evolutionary divergence between IF and lamin genes. This I expect to become more obvious once the entire lamin gene is known.

\section{Max-Planck Institute for \\ Biophysical Chemistry, \\ D-34 Goettingen, FRG}

MeKeon, F.D., Kirschner, M.W. \& Caput, D. Nature 319, 463-468 (1986)

Steinert, P.M., Steven, A.C. \& Roop, D.R. Cell 42, 411-419 (1985).

3. Karn, J. Brenner, S. \& Barnett, L. Proc. natn. Acad. Sci. U.S.A. 80, 4253-4257 (1983).

\section{The mechanics of visual acuity}

SIR-Anyone who needs strongish (positive) reading glasses but does not suffer unduly from astigmatism can see that visual acuity is not just a function of eye lens quality plus receptor spacing. All that is needed is a red-light-emitting diode behind a square aperture. Looking at this without glasses produces not a single blurred image but an array of about eight red squares, more or less sharply defined. A 'perfect' eye would presumably produce a completely regular array; mine do not, and differ individually.

It follows that the eye-cortex combination acts holographically, in that separate portions of a blurred image give rise to the impression of a sharp image. Furthermore, if instead the image of a point source is properly focused, the system can be expected to give rise to a visual acuity greater than indicated by optical considerations alone.

The compound eyes of insects are known to act in a similar manner, because individual images are too poor to account for bees' discriminatory power, for example. So it is perhaps not surprising that the larger animal eye, producing good optical images, should nevertheless have retained the image-processing power of the cortex representing an earlier stage in the evolution of the eye.

Bower Chalke,

T. NASH

Salisbury, Wilts SP5 5BW, UK

\section{The human factor in} mammoth extinction

Sir-A recent News and Views piece by Diamond ${ }^{1}$ discusses mammoth hunting and extinction. While I agree that sophisticated hunting techniques must have been available to palaeolithic mammoth hunters, close spearing or hamstringing, as practised by modern Pygmy elephant hunters, is dependent on the possession of razor-sharp iron spearheads ${ }^{2}$; and the existence of a pharmacopoeia of poisons among Palaeolithic hunters is of course undocumented. Trunk snares or weighted hanging spears would seem to depend on the prey moving among rather heavy forest vegetation, whereas the stomach contents of mammoths attest to their inhabiing sparsely forested, open, steppelike grasslands. Palaeolithic peoples probably trapped mammoths with fire or with footsnares and pitfalls set or dug along regularly used routes. As zoo curators know, a fall of only a metre or even a stumble may be potentially fatal to a large mammal, so a footsnare or pitfall would have been a relatively safe and effective aid to mammoth hunting.

Nevertheless, contrary to Diamond's contention, French and Spanish cave paintings do not unequivocally show these hunting techniques. Tectiform signs in Palaeolithic art are certainly sometimes interpreted as depicting traps and pitfalls, but the interpretation of these signs is subject to debate. They have also been viewed as depicting shelters or maps, or as having a more esoteric symbolic significance.

Finally, Diamond concludes that mammoth extinction is probably the direct result of the proficiency of human hunting. However, the most recent compendium of explanations $^{3}$ for Pleistocene megafauna extinctions appears to favour ecological or climatic causes. Certainly the Pleistocene overkill hypothesis originally elaborated by P.S. Martin has not been accepted without debate, and may have lost some potency as an explanatory hypothesis during the past twenty years, even with the introduction of the concept of a blitzkrieg overkill which might explain the paucity of archaeological evidence.

Susan CACHEL

Douglas College, Rutgers University, New Brunswick, New Jersey 08903, USA

1. Diamond, J. Nature 319, 265-266 (198)

. Coon, C.S. The Hunting Peoples (Little, Brown, Boston 1971).

. Martin, P.S. \& Klein, R.G. Quaternary Extinctions (University of Arizona Press, Tucscon, 1984)

Scientific Correspondence is intended to provide a forum in which readers may raise points of a rather technical character which are not provoked by articles or letters previously published. 